\title{
Malignant Eyelid Neoplasm
}

National Cancer Institute

\section{Source}

National Cancer Institute. Malignant Eyelid Neoplasm. NCI Thesaurus. Code C6786.

A primary or metastatic malignant neoplasm that affects the eyelid. 